\title{
Feedback of kinetic energy into the IGM by supermassive black holes
}

\author{
George Chartas ${ }^{1}$, W. N. Brandt ${ }^{1}$ \\ and S. C. Gallagher ${ }^{2}$ \\ ${ }^{1}$ Department of Astronomy \& Astrophysics, Pennsylvania State University, University Park, \\ PA 16802, USA email:chartas@astro.psu.edu, niel@astro.psu.edu \\ ${ }^{2}$ University of California, Los Angeles, Division of Astronomy \& Astrophysics, Los Angeles, \\ CA 90095, USA email:sgall@astro.UCLA.EDU
}

\begin{abstract}
Our recent Chandra and XMM-Newton observations of two high redshift broad absorption line (BAL) quasars have revealed the presence of massive outflows of highly ionized, high-metallicity material driven from near the black hole (a few gravitational radii) with velocities of up to $0.4 c$. The inferred mass outflow rates of $1-10 M_{\odot} \mathrm{yr}^{-1}$ and the measured outflow velocities, that significantly exceed the escape velocities of galaxies and clusters of galaxies, imply that quasar winds provide an important feedback mechanism for kinetic energy injection into the IGM. These quasar outflows may also play an important role in the coevolution of black holes and their host galaxies. We present new constraints on the location of the X-ray BAL material based on our analysis of the variability of the ionization properties of the X-ray absorbers. The location and ionization properties of the X-ray BAL material are crucial in inferring the mass outflow rate and rate of kinetic energy injected into the IGM.
\end{abstract}

\section{Introduction}

With the advent of XMM-Newton and Chandra it has become possible to infer the kinematic and ionization properties of highly ionized X-ray absorbers in Seyfert 1 galaxies (e.g., Crenshaw et al. 1999, 2003; Kraemer et al. 2001; Sako et al. 2001; Kaspi et al. 2002; Netzer et al. 2003; Crenshaw et al. 2003). Because of the heavy X-ray absorption in broad absorption line (BAL) quasars, the X-ray spectra obtained for them to date have modest (e.g., PG 2112+059) to poor signal-to-noise ratio $(\mathrm{S} / \mathrm{N})$ and cannot accurately constrain the column densities, ionization states, geometry, or kinematic properties of Xray BALs. (e.g., Green et al. 2001; Gallagher et al. 2001, 2002). Recent XMM-Newton and Chandra observations of the gravitationally lensed (GL) BAL quasars PG 1115+080 and APM $08279+5255$ have provided new insights into the structure of quasar outflows and the enrichment of the interstellar medium (ISM) and the intergalactic medium (IGM) by quasar winds (Chartas et al. 2002, 2003). This was suggested by the identification of broad $\mathrm{X}$-ray absorption lines at rest-frame energies of 8.1 and $9.8 \mathrm{keV}$ for APM $08279+5255$ and 7.4 and $9.5 \mathrm{keV}$ for PG 1115+080. By interpreting these absorption lines as resonant absorption lines of highly ionized $\mathrm{Fe}$ (e.g., Fe XxV $\mathrm{K} \alpha$ ), we inferred the presence of X-ray BAL material outflowing from the central source at velocities of $\sim 0.2 c$ and $\sim$ $0.4 c$ for $\mathrm{APM} 08279+5255$ and $\sim 0.1 c$ and $\sim 0.3 c$ for $\mathrm{PG} 1115+080$. One of the key implications of the Chartas et al. $(2002,2003)$ results is that X-ray BAL material appears to be located at smaller radii than the UV BAL region (see Figure 1) and could represent the shielding gas proposed in several theoretical studies of line-driven disk winds (e.g., Murray et al. 1995; Proga et al. 2000). The confirmation of relativistic outflows in most quasars would imply that these energetic winds have a significant impact in regulating the growth of the black hole, perhaps explaining the $M_{B H^{-}} \sigma$ relation, and halting star 
formation and shaping the evolution of their host galaxies. The importance of the quasar feedback mechanism has been recently demonstrated in theoretical models of structure formation that incorporate the effects of quasar outflows [e.g., Scannapieco \& Oh 2004 (SO04); Granato et al. 2004 (GR04)].

The main question addressed in this paper is the following: How important are quasar outflows in regulating the growth of black holes and in contributing to the enrichment of the IGM? We address this question in $\S 2$ by discussing the frequency of quasar outflows, by presenting interpretations of the BAL quasar phenomenon, and by providing estimates of the mass outflow rate and rate of kinetic energy injection into the IGM for the case of APM $08279+5255$. In $\S 3$ we review recent studies that incorporate quasar feedback using constraints provided from recent observations [including constraints presented in Chartas et al. (2002)] in modeling the evolution of quasars and their host galaxies. Finally, in section $\S 4$ we summarize our results.

\section{How Important is the Quasar Feedback Process?}

Observations of absorption lines in BAL quasars provide the following constraints on quasar outflows: the frequency of outflows in quasars, their launching locations and driving mechanisms, their kinematic and ionization properties, and their covering factors. These constraints are used to derive estimates of the mass outflow rates and rates of kinetic energy injected into the IGM. In the following subsections we briefly describe these constraints and provide an estimate for the mass outflow rate and rate of kinetic energy injection for the BAL quasar APM 08279+5255.

\subsection{Frequency of Outflows in Quasars}

The importance of outflows in regulating the growth of black holes and providing a feedback mechanism for kinetic energy injection into the IGM depends in part on the frequency of these outflows in quasars. In a model suggested by Weymann et al. (1991), winds exist in most quasars; however, because of the relatively small opening angles of these outflows only a small fraction of radio-quiet quasars have detectable BAL features in their UV and/or optical spectra. Spectropolarimetric observations, the statistics of gravitationally lensed BAL quasars, improved assessments of incompleteness, and theoretical arguments suggest that present optical surveys may be biased against the detection of BAL quasars (e.g., Goodrich 1997; Krolik \& Voit 1998; Chartas 2000; Tolea, Krolik, \& Tsvetanov 2002; Hewett \& Foltz 2003). These analyses indicate that the true fraction of BAL quasars and BAL covering factors may be substantially larger ( $\gtrsim 20 \%)$ than the often quoted value of $\sim 10 \%$. Recent observations of eight quasars discovered by the Sloan Digital Sky Survey with redshifts ranging between 4.9 and 6.4 indicate that the fraction of BAL quasars at high redshift may be as large as 50\% (Maiolino et al. 2003). Because of the limited number of quasars studied in detail in this redshift range the result is still considered preliminary, and a larger sample is needed to confirm the evolution of the frequency of BAL quasars with redshift. Other interpretations of the BAL quasar phenomenon include a proposal that BAL quasars are a stage in the life cycle of quasars (e.g., Briggs et al. 1984) and the idea that absorbing outflows occur in only a fraction of quasars with high accretion rates (Becker et al. 2000).

\subsection{Location and Driving Mechanisms}

The variability of the X-ray BALs observed in APM $08279+5255$ indicates that these absorbers are most likely launched at $r<10^{16}\left(M_{B H} / M_{8}\right)^{1 / 2} \mathrm{~cm}$. The driving mechanism 
for the UV gas is thought to be scattering in atomic resonance lines; however, the driving mechanism for highly ionized X-ray gas is not well understood.

\subsection{Kinematic and Ionization Properties}

The spectrum of APM $08279+5255$ indicates the presence of complex low-energy absorption in the $0.5-1.0 \mathrm{keV}$ observed energy band and high-energy absorption in the 1.5-3 keV observed energy band. By interpreting the high-energy absorption features in the Chandra observation of APM $08279+5255$ as resonant absorption lines of highly ionized Fe (Fe Xxv K $\alpha$ ), we inferred the presence of X-ray BAL material outflowing from the central source at velocities of $\sim 0.2 c$ and $\sim 0.4 c$. Our multiple resonant absorption-lines model provides acceptable fits to both Chandra and XMM-Newton spectra and implies a velocity and width change of the X-ray BAL components between the two observations. From a simple curve-of-growth analysis we estimated that the total hydrogen column densities corresponding to the absorption lines at rest energies of $8.1 \mathrm{keV}$ and $9.8 \mathrm{keV}$ are $N_{\mathrm{H}}=4 \times 10^{22} \mathrm{~cm}^{-2}$ and $N_{\mathrm{H}}=4 \times 10^{23} \mathrm{~cm}^{-2}$, respectively. Our best-fit ionization parameter for the low-energy absorption was $\xi=20_{-20}^{+70} \mathrm{erg} \mathrm{cm} \mathrm{s}^{-1}$, whereas, the presence of highly ionized Fe (high-energy absorption lines) requires $\xi \gtrsim 1000 \mathrm{erg} \mathrm{cm} \mathrm{s}^{-1}$.

\subsection{Mass Outflow Rate and Kinetic Energy Injection}

Our constraints on the velocity, column density and location of the BAL material allow us to estimate the mass outflow rate from the expression $\dot{M}=4 \pi r(r / \Delta r) N_{\mathrm{H}} m_{p} v_{\text {wind }} f_{c}$. For the case of APM $08279+5255$ we find $\dot{M} \sim 1-10 M_{\odot} y r^{-1}$ (see Figure 1). This outflow rate is comparable to the accretion rate in APM $08279+5255$ which we estimate to be $1.8 \times 10^{-3}\left(L_{44} / \eta\right) M_{\odot} \mathrm{yr}^{-1} \sim 10-40 M_{\odot} \mathrm{yr}^{-1}$. One of the goals of UV and Xray observations of AGN outflows is to constrain the fraction $\epsilon_{k}$ of the total bolometric energy released over a quasar's lifetime into the ISM and IGM in the form kinetic energy injection. For the case of APM $08279+5255$ we estimate that $\epsilon_{k}=0.02-0.22$.

\section{Models of Structure Evolution that Incorporate Quasar Feedback}

Recent models of structure formation incorporate the effects of quasar outflows (SO04; GR04).A basic assumption in the models of SO04 is that all quasars host outflows. SO04 find that by choosing $\epsilon_{k} \sim 0.05$ they can successfully model the observed evolution of the $B$-band quasar luminosity function between redshifts of 0.25 and 6.25 . Their adopted value of an average efficiency for quasar outfllows of $\epsilon_{k} \sim 0.05$ is consistent with our estimated value based on the Chandra observations of APM $08279+5255$. They conclude that quasars heat the IGM above a critical entropy level preventing cooling and thus regulating their own formation. In a related study, the results from our analysis of the quasar outflow in APM $08279+5255$ have been incorporated in a physical model for the coevolution of quasars and their hosts (GR04). In particular, GR04 find that the quasar feedback process can heat the ISM gas and unbind it. The gas can then flow out of the host galaxy, thus inhibiting star formation and the growth of the black hole itself.

\section{Conclusions}

We have detected relativistic outflows $(v=0.1-0.4 c)$ of highly ionized gas in two distant quasars. The mass outflow rate in APM $08279+5255\left(\dot{M} \sim 1-10 M_{\odot} y r^{-1}\right)$ is found to be comparable to its accretion rate and the fraction of bolometric energy released in the form of kinetic energy is $\epsilon_{K} \sim 0.02-0.22$. The confirmation of relativistic outflows in most 

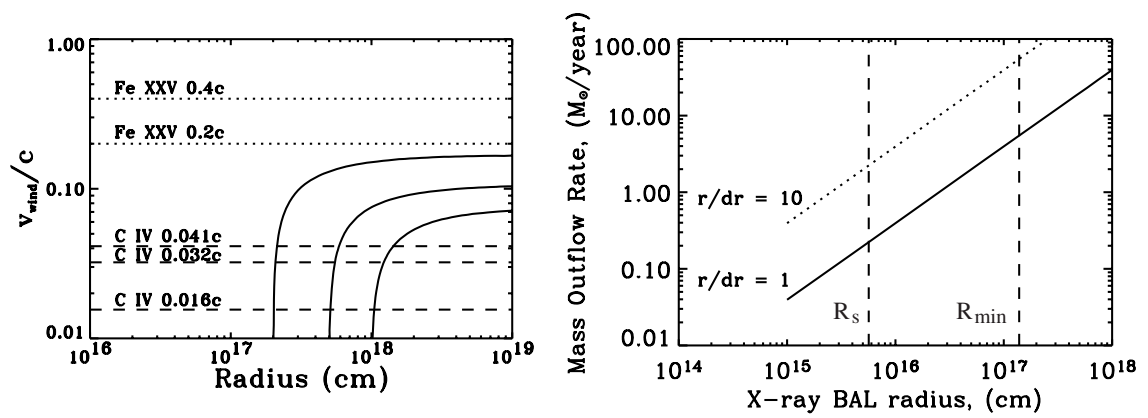

Figure 1. (left) Wind velocity as a function of radius from the central source for a radiation-pressure driven wind. For a qualitative comparison we have estimated the wind velocities for launching radii of $2 \times 10^{17} \mathrm{~cm}, 5 \times 10^{17} \mathrm{~cm}$, and $1 \times 10^{18} \mathrm{~cm}$. We have over-plotted the observed C IV BAL (dashed lines) and Fe XXV BAL (dotted lines) velocities. (right) Estimate of mass outflow rate versus radius for APM $08279+5255 . R_{s}$ and $R_{m i n}$ are the Schwarzschild and the minimum launching radii, respectively.

quasars would imply that quasar outflows have a significant impact in inhibiting their own growth, halting star formation and shaping the evolution of their host galaxies.

\section{Acknowledgements}

We acknowledge financial support from NASA grant NAG5-9949. WNB acknowledges financial support from NASA grant NAG5-9932. Support for SCG was provided by NASA through the Spitzer Fellowship Program, under award 1256317.

\section{References}

Becker, R. H., White, R. L., Gregg, M. D., Brotherton, M. S., Laurent-Muehleisen, S. A., \& Arav, N. 2000, ApJ, 538, 72

Briggs, F. H., Turnshek, D. A., \& Wolfe, A. M. 1984, ApJ, 287, 549

Chartas, G. 2000, ApJ, 531, 81

Chartas, G., Brandt, W. N., \& Gallagher, S. C. 2003, ApJ, 595, 85

Chartas, G., Brandt, W. N., Gallagher, S. C., \& Garmire, G. P. 2002, ApJ, 579, 169

Crenshaw, D. M., Kraemer, S. B., Boggess, A., Maran, S. P., Mushotzky, R. F., \& Wu, C. 1999, ApJ, 516, 750

Crenshaw, D. M., et al. 2003, ApJ, 594, 116

Gallagher, S. C., Brandt, W. N., Laor, A., Elvis, M., Mathur, S., Wills, B. J., \& Iyomoto, N. 2001, ApJ, 546, 795

Gallagher, S. C., Brandt, W. N., Chartas, G., \& Garmire, G. P. 2002, ApJ, 567, 37

Goodrich, R. W. 1997, ApJ, 474, 606

Granato, G. L., De Zotti, G., Silva, L., Bressan, A., \& Danese, L. 2004, ApJ, 600, 580

Hewett, P. C. \& Foltz, C. B. 2003, AJ, 125, 1784

Kaspi, S., et al. 2002, ApJ, 574, 643

Kraemer, S. B., et al. 2001, ApJ, 551, 671

Krolik, J. H. \& Voit, G. M. 1998, ApJ, 497, L5

Netzer, H., et al. 2003, ApJ, 599, 933

Maiolino, R., et al. 2003, (astro-ph/0312402)

Murray, N. \& Chiang, J. 1995, ApJ, 454, L105

Proga, D., Stone, J. M., \& Kallman, T. R. 2000, ApJ, 543, 686

Sako, M., et al. 2001, A\&A, 365, L168

Scannapieco, E.,\& Oh, S. P. 2004 (astro-ph/040187)

Tolea, A., Krolik, J. H., \& Tsvetanov, Z. 2002, ApJ, 578, L31

Weymann, R. J., Morris, S. L., Foltz, C. B., \& Hewett, P. C. 1991, ApJ, 373, 23 